\title{
Prognosis of Conservative Management of Shoulder Dislocation Fracture: A Case Report
}

\author{
Tanya Gujral ${ }^{1}$, Mohd. Asif ${ }^{2}$ \\ ${ }^{1}$ BPT Scholar, Department of Rehabilitation Sciences, HIMSR, Jamia Hamdard, New Delhi \\ ${ }^{2}$ Assistant Professor, Physiotherapy Department, SHS, NIU, Greater Noida
}

\begin{abstract}
The Gleno-humeral joint is a supreme joint of the shoulder complex. In all major joints of the human body, gleno-humeral joint is the most common dislocating joint. Around $98 \%$ of the shoulder evicts in the anterior and about $2 \%$ in the posterior direction. Traumatic shoulder dislocations occur may be due to a forceful collision, rapid jerky movement, falling on an outstretched arm and Atraumatic due to repetitive microtrauma. Isolated fractures of greater tuberosity are exceptional injuries, but it may also see in combination with anterior shoulder dislocation approximately $15 \%$ to $30 \%$. Conservative management of anterior shoulder dislocation with displaced greater tuberosity fracture has poor results and also a greater chance of nonunion, malunion and osteonecrosis. Operative management had good results and allows to take part in early rehabilitation. In our case a 30 year old male injured his left shoulder. Radiographs of the shoulder confirmed anterior dislocation of shoulder with greater tuberosity fracture. The patient was managed by, Open Reduction Internal Fixation and intramedullary nail fixation under general anesthesia. After surgery patient manage by passive, active assistive, close chain and strengthening exercises. Slings were used for supporting the shoulder joint and cryotherapy used for reduction of pain and swelling.
\end{abstract}

Keywords: Gleno-humeral joint, Traumatic, Anterior dislocation, Fracture, ORIF

\section{Introduction}

The Gleno-humeral joint is a supreme joint of the shoulder complex. Itis composed by the articulation between the humeral head and the lateral scapula. Shoulder stability is the result of a compositesynergy between static and dynamic shoulder controls[1].

In all major joints of the human body, gleno-humeral joint is the most common dislocating joint, approximately $98 \%$ of the shoulder evicts in the anterior direction[2-4] and about $2 \%$ in the posterior direction[2]. Shoulder dislocation may be due to traumatic $(96 \%)$ or atraumatic (4\%)events [5]. Traumatic shoulder dislocations occurs may be due to a forceful collision, rapid jerky movement,falling on an outstretched arm and Atraumatic due to repetitive microtrauma [6]. Isolated fractures of greater tuberosity are exceptional injuries, i.e.(GT) comprise $17 \%$ to $21 \%$ of proximal humeral fracturesbut also seen in combination with anterior shoulder dislocationapproximately $15 \%$ to $30 \%[7]$.

Neer[8] was concludedthatconservative management of anterior shoulder dislocation with displaced greater tuberosity fracture haspoor results and also decrease osteosynthesis. He also founded that greater chanceof nonunion, malunion and osteonecrosis of the humeral head. Operative management had good results and allows to take part in early rehabilitation [6]. Early rehabilitation helps in the earlier rebound to thework or sports. This case of a young male with anterior traumatic shoulder dislocation with displaced greater tuberosity fracture and its rehabilitation.

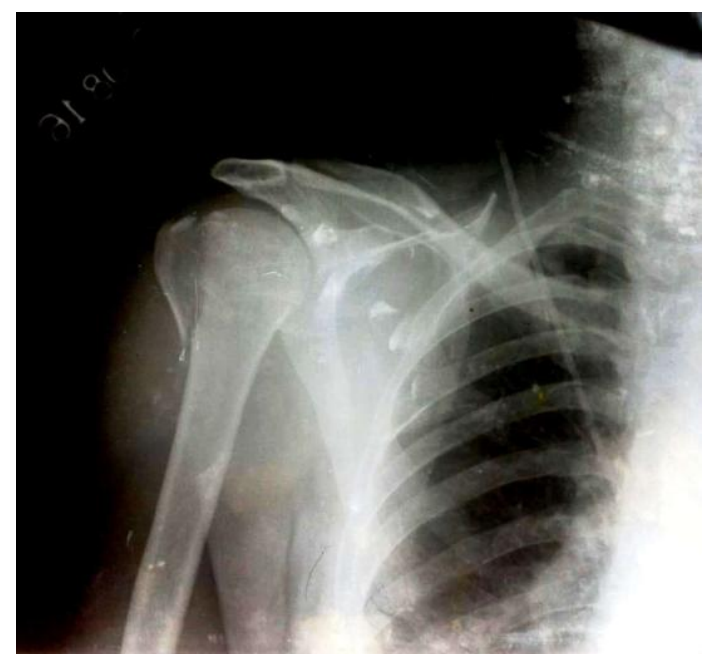

Figure 1: Preoperative X-ray (AP view)

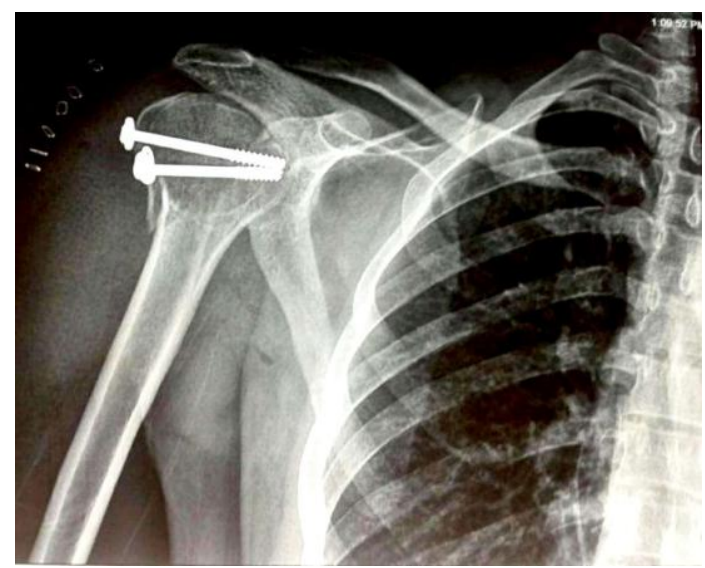

Figure 2: Postoperative X-ray (AP view)

\section{Case Report}

A 30 year old male injured his left shoulder during a fall from a ladder. The patient developed severe pain and was 


\section{International Journal of Science and Research (IJSR) \\ ISSN (Online): 2319-7064}

Index Copernicus Value (2015): 78.96 | Impact Factor (2015): 6.391

unable to move his left upper extremity. Immediately following the injury, the patient supported the injured extremity in a position of adduction and internal rotation as it decreased his pain. He reported to the hospital with complaints of pain, swelling, deformed shoulder and loss of shoulder movements. During the initial examination, humeral head was palpable anteriorly, rounded contour of the shoulder (anterolaterally) was tender on palpation but neurovascular status was intact. The anteroposterior radiographs [Figure 1] of the shoulder confirmed anterior dislocation of shoulder with greater tuberosity fracture. The patient was managed by, Open Reduction Internal Fixation (ORIF) and cancellous screws under general anesthesia [Figure 2]. The limb was immobilized in adduction and internal rotation for 6 weeks and was referred for physical therapy after 4 weeks.

The initial physical examination during the first day at the rehab center revealed decreased shoulder strength, absent arm swinging, protracted and elevated left shoulder and the movements of the wrist and elbow were within complete range of motion. The goals of physical therapy program were as follows: Immediate goals - reduced pain, maintain the available range of motion, protection from re- injury, prevent disuse atrophy. Long term goals - increase range of motion, improved strength and flexibility and an early return to functional activities.

\section{Rehabilitation Program}

After the cause of the instability had been evaluated and classified, the patient began performing a specific set of exercises designed to strengthen the deltoid and the muscles of the rotator cuff. It is based on the principle of progressive resistance.

\section{Phase 1: Weeks 0-2}

Restrictions:-Avoiding provocative positions of the shoulder that increase the risk of recurrent instability (External rotation, Abduction and Distraction) .

At shoulder joint:- Initially for first two weeks, active assisted movements of glenohumeral joint along with close chain isometrics strengthening of scapulothoracic and humeral muscles were made to perform by te therapist to the patient within the sling. Cryotherapy in the postoperative shoulder (applied for 15-minute durations every 1 to 2 waking hours for the first 24 hours, and 4 to 6 times daily for the ensuing 9 days) has been shown to significantly decrease the frequency and intensity of shoulder pain both at rest and during rehabilitation.

At elbow joint:- Passive motion at elbow joint was performed by the patient, which was progressed to active motion at the end of the $1^{\text {st }}$ week and Pronation and supination as tolerated.

Muscle Strengthening:- Scapular stabilizer strengthening and Grip strengthening.

Phase 2: During $3^{\text {rd }}$ to $4^{\text {th }}$ week

Criteria for Progression to Phase2:- Reduced pain and tenderness
Restrictions:-Avoid provocative positions of the shoulder that risk recurrent instability.

\section{Motion: shoulder}

Goals

- 140 degree of forward flexion

- 40 degrees of external rotation with the arm at the Pain Control side .

$\underline{\text { Exercises }}$

- The sling was discontinued

- Codaman pendulum exercises

- Active assisted movement of glenohumeral joint within pain free limit.

- Progressive resisted exercises for shoulder complex muscles using slight manual resistance within pain free range.

- Scapular retraction (rhomboideus, middle trapezius). Scapular protraction (serratus anterior).

- Scapular depression (latissimus dorsi, trapezius,serratus anterior).

- External rotation.

- Shoulder shrugs (trapezius, levator scapulae).

Phase 3: During $5^{\text {th }}$ to $7^{\text {th }}$ week

Motion: Shoulder

- Active movements of glenohumeral joint within pain free range.

- Stretching of inferior and posterior capsule.

- Exercises were performed through an arc of 45 degree in each of the five planes of motion.

- Strengthening of scapular stabilizers by theraband.

Reevaluation after 6 weeks reveals increase in range of motion with no pain and decreased apprehensions.

Phase 4: During $8^{\text {th }}$ to 10 th $^{\text {th }}$ week

- Open kinematic chain Scapular strengthening using dumbbell.

- Initiate deltoid strengthening in the plane of the scapula to 90 degrees of elevation.

- Open chain Rotator cuff strengthening using Theraband.

- Incorporated endurance training for the upper extremity upper body Ergometer.

- Proprioceptive training (catching and throwing activities).

Home exercise program comprising of

- Range of motion exercises twice a week

- Gleno-humeral and scapular strengthening thrice a week with self-stretching of inferior and posterior capsule was prescribed.

\section{Results}

It is very important that the exercise protocol is continued for atleast twice to thrice a week once the stability of the shoulder has been achieved through the rehabilitation program. The degree of compliance with the rehabilitation program was measured by the ability of the patient to demonstrate the exercise routine accurately, recall which color of therabands indicated strongest or weakest, and state 


\section{International Journal of Science and Research (IJSR) \\ ISSN (Online): 2319-7064 \\ Index Copernicus Value (2015): 78.96 | Impact Factor (2015): 6.391}

which exercise was the most difficult to perform. Overall, the patient had a good result after the exercise regime. Maximum stability of the shoulder was achieved after 10 weeks.

\section{Discussion}

The shoulder is a joint evolved for mobility and to some extent, stability has been sacrificed to achieve a wide range of motion[1]. The shoulder complex comprises of three major joints: the glenohumeral, acromioclavicular, and sternoclavicular. The glenohumeral joint is an articulation between the humeral head and the lateral scapula. Stability of the joint is dependent upon several factors that may be classified as static and dynamic restraints. Static stabilizers are the labrum, negative intra articular pressure, the articular surface of the glenoid and the joint capsule with its ligaments. The dynamic stabilizers are the rotator cuff muscles, biceps, pectorals, biceps and peri-articular muscles[2].

Isolated fractures of greater tuberosity are rare injuries, but can be seen in conjugation with $5-15 \%$ of anterior shoulder dislocation [2,7]. In up to $98 \%$ of cases, the shoulder displaces in an anterior direction[2,3,4] and in about $2 \%$ of cases it is displaced in the posterior direction[2].

The most frequent mechanism of anterior shoulder dislocation has been characterized as forced external rotation and abduction of the humerus as seen in a basketball player who attempts to block an overhead pass[4,6]. Other mechanisms of injury have included a fall onto an elevated out- stretched arm and direct force application to the posterior aspect of the humeral head restraint $[4,6]$. Proprioceptive loss have been exhibited for patients with traumatic anterior shoulder instability $[9,10]$.

The basic aim of treating shoulder dislocations whether conservatively or surgically is to restore the normal anatomical and biomechanical articulating surfaces. For this reason, each patient who has instability of the shoulder should be thoroughly evaluated if a successful result of treatment is to be expected. Operative intervention is not a new concept. Yoneda et al reported that, in 104 patients who had a traumatic anterior dislocation, a satisfactory result was achieved in 82.7 percent after use of a program of exercises for the shoulder[11].

Rehabilitation is the key whether managed surgically or conservatively. It involves active and active assisted range of motion exercises started initially in the pain free range and progressing gradually. The later phases involve strengthening of scapular musculature and developing endurance for the entire upper extremity. On achieving full pain free range, exercises were modified to ensure an early and safe return to activities of daily living.

\section{Conclusion}

There have been significant advances in methods to restore function in unidirectional shoulder instability. Biomechanical and anatomical studies have enhanced our understanding of the mechanics of the shoulder joint with intimation for the management and rehabilitation of shoulder instability. Further investigation of the mechanisms responsible for the dynamic glenohumeral control will help to delineate the optimal treatment for shoulder instability.

\section{Conflict of Interest}

There is no conflict of interest to declare.

\section{Refrences}

[1] Moseley HF. Recurrent Dislocation of the Shoulder. London, England: E and S Livingstone Ltd.; 1961

[2] Rowe CR. Prognosis in dislocations of the shoulder. J Bone Joint Surg. 1956;38A:957-977

[3] Burkhead WZ, Jr., Rockwood CA, Jr. Treatment of instability of the shoulder with an exercise program. J Bone Joint Surg Am. 1992;74:890-896

[4] Liu SH, Henry MH. Anterior shoulder instability. Current review. Clin Orthop. 1996;327-337

[5] Rehabilitation for shoulder instability. A Jaggi and S Lambert.

[6] Aronen JG, Regan K. Decreasing the incidence of recurrence of first time anterior shoulder dislocations with rehabilitation. Am J Sports Med.

[7] Lind T, Kroner K, Jensen J. The epidemiology offractures of the proximal humerus. Arch Orthop Trauma Surg. 1989;108:285-287

[8] Neer CS 2nd. Displaced proximal humeral fractures. II. Treatment of three-part and four-part displacement. J Bone Joint Surg Am 1970;52:1090-103.

[9] Smith RL, Brunolli J. Shoulder kinesthesia after anterior glenohumeral joint dislocation. Phys Ther. 1989;69:106-12

[10] Warner JJ, Lephart S, Fu FH. Role of proprioception in pathoetiology of shoulder instability. Clin Orthop. 1996;35-39.

[11] Yoneda M, Izawa K, Hirooka A, et al. Indicators of superior glenoid labral detachment on magnetic resonance imaging and computed tomography arthrography. J Shoulder Elbow Surg. 1998 Jan-Feb. 7(1):2-12. [Medline].

\section{Author Profile}

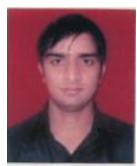

Dr. MHD. ASIF (PT), (Corresponding Author) Assistant Professor, Physiotherapy Department, SHS, NIU, Greater Noida.

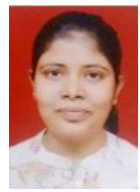

Miss. Tanya Gujral is BPT Scholar, Department of Rehabilitation sciences, HIMSR, Jamia Hamdard, New Delhi. 